\title{
Inhibition of angiogenesis and suppression of colorectal cancer metastatic to the liver using the Sleeping Beauty Transposon System
}

\author{
Lalitha R Belur ${ }^{1,2,3^{*}}$, Kelly M Podetz-Pedersen ${ }^{1,2,3}$, Brent S Sorenson ${ }^{4}$, Alice H Hsu ${ }^{1,3}$, Josh B Parker ${ }^{4}$,
} Cathy S Carlson ${ }^{4}$, Daniel A Saltzman ${ }^{5}$, S Ramakrishnan ${ }^{6}$, R Scott Mclvor ${ }^{1,2,3^{*}}$

\begin{abstract}
Background: Metastatic colon cancer is one of the leading causes of cancer-related death worldwide, with disease progression and metastatic spread being closely associated with angiogenesis. We investigated whether an antiangiogenic gene transfer approach using the Sleeping Beauty (SB) transposon system could be used to inhibit growth of colorectal tumors metastatic to the liver.

Results: Liver CT26 tumor-bearing mice were hydrodynamically injected with different doses of a plasmid containing a transposon encoding an angiostatin-endostatin fusion gene (Statin AE) along with varying amounts of SB transposase-encoding plasmid. Animals that were injected with a low dose $(10 \mu \mathrm{g})$ of Statin AE transposon plasmid showed a significant decrease in tumor formation only when co-injected with SB transposase-encoding plasmid, while for animals injected with a higher dose $(25 \mu \mathrm{g})$ of Statin AE transposon, co-injection of SB transposase-encoding plasmid did not significantly affect tumor load. For animals injected with $10 \mu \mathrm{g}$ Statin AE transposon plasmid, the number of tumor nodules was inversely proportional to the amount of co-injected SB plasmid. Suppression of metastases was further evident in histological analyses, in which untreated animals showed higher levels of tumor cell proliferation and tumor vascularization than animals treated with low dose transposon plasmid.
\end{abstract}

Conclusion: These results demonstrate that hepatic colorectal metastases can be reduced using antiangiogenic transposons, and provide evidence for the importance of the transposition process in mediating suppression of these tumors.

\section{Background}

Carcinoma of the colon is the second most common cause of cancer-related death in the United States and other developed countries [1]. The primary cause of mortality is dissemination of the disease to secondary sites, with the liver being the primary, and most critical, organ for development of metastasis [2,3]. Liver resection is the only effective treatment to facilitate a potential cure. However, less than $10 \%$ of patients are eligible for surgery, since they present with advanced or disseminated disease due to the absence of early diagnostic symptoms [2-4].

\footnotetext{
* Correspondence: belur001@umn.edu; mcivo001@umn.edu

${ }^{1}$ Center for Genome Engineering, University of Minnesota, Minneapolis, MN 55455, USA

Full list of author information is available at the end of the article
}

Tumor neovascularization plays a critical role in colorectal cancer progression, and increased angiogenesis has been associated with poor prognosis and relapse of colorectal disease $[5,6]$. There are several small molecule inhibitors of angiogenesis currently in clinical trials [7]. The anti-VEGF antiangiogenic antibody bevacizumab is now used clinically as a first line treatment in combination with standard first and second-line chemotherapy regimens for treatment of metastatic colorectal cancer, conferring a significant increase in survival time (20-25 months) [8,9]. However, antiangiogenic factors have a cytostatic rather than cytotoxic effect, therefore requiring continuous and possibly lifelong administration of the recombinant protein $[10,11]$. Introduction of sequences encoding antiangiogenic gene products is an alternate approach to achieve continuous and sustained expression of angiostatic factors

\section{() Biomed Central}


in neoplastic tissue, thus counteracting tumor-induced angiogenesis.

Both viral and non-viral vector systems have been tested for potential therapeutic gene transfer against colorectal cancer. Viral vectors have been used by most investigators for gene delivery, due to the higher efficiency of gene transfer compared to non-viral systems. Viral vector types that have been used to deliver antiangiogenic genes for therapy of colorectal cancer include adenoviral vectors [12-15] and adeno-associated viral (AAV) vectors [16], and non-viral vectors include HVJ cationic liposomes and naked plasmid DNA. HVJ-cationic liposomes were shown to be effective in inhibiting angiogenesis by repeat intratumoral injections of vector encoding mouse macrophage metalloelastase in a subcutaneous model of colorectal cancer [17]. Uesato et al expressed angiostatin and endostatin in subcutaneous tumors after repeated low-voltage electroporation and achieved decreased tumor growth [18]. More recently, Wen et al reported hydrodynamic plasmid injection to express NK4 in a hepatic model of liver metastasis, with successful inhibition of tumor formation $[19,20]$. Nonviral anti-angiogenic gene delivery has thus, been used successfully, with therapeutic benefits in inhibiting the growth of colorectal tumors, but the duration of effectiveness is constrained by the transient period of gene expression.

The Sleeping Beauty (SB) transposon system combines the advantages of non-viral plasmid-based vector systems with the integrative capabilities of some viral vectors. This plasmid-based vector system provides prolonged expression of the transgene through integration into the host chromosome, thereby circumventing the need for repeated administration of the therapeutic gene [21]. The SB transposon system has been successfully used to transfer genes into a variety of cell types [22-25], including neoplastic tissue [26-28]. This system consists of 2 components; a transposon, comprising a gene of interest flanked by indirect repeat sequences, and the synthetic SB transposase, which catalyzes excision and integration of the gene into genomic DNA.

In the present study, the SB transposon system was used to achieve transfer of antiangiogenic genes into tumor-bearing animals. We investigated the antitumor effects of a transposon vector that encodes an angiostatin-endostatin fusion gene (Statin AE), administered in a CT26 mouse model of colorectal cancer metastatic to the liver. Statin AE transposon administration was associated with a significant antitumor effect as gauged by inhibition of tumor growth, and reduction in tumor vasculature. A dose-dependent requirement for SB transposase-encoding plasmid at lower doses of Statin AE transposon was observed, implicating the importance of transposition and stable Statin AE expression in low substrate (transposon) conditions such as those likely to be achieved in large animals or humans. These results demonstrate the potential effectiveness of the SB transposon system for therapeutic antiangiogenic gene transfer in metastatic colorectal cancer.

\section{Results \\ Hepatic Gene Delivery and Localization of Gene Expression}

There have been several studies reported in which vectors containing either angiostatin or endostatin resulted in significant tumor regression upon administration to tumor bearing mice. In addition, combined treatment of tumor bearing mice with vectors encoding both angiostatin and endostatin resulted in an additive effect in tumor growth inhibition when compared to treatment using either of these agents alone [29-31]. The StatinAE gene, which is a fusion of murine angiostatin and endostatin genes, has been demonstrated to confer significant inhibition of subcutaneously implanted human glioblastoma and melanoma xenografts $[27,32]$. We therefore constructed a Sleeping Beauty transposon plasmid containing the StatinAE and firefly luciferase genes regulated by a bidirectional promoter (pKT2/BidEAL, Figure 1A). In this construct a luciferase transgene is transcriptionally regulated by the phosphoglycerate kinase (PGK) promoter, while in the opposite orientation a CpG free enhancer/elongation factor 1-alpha (EF1- $\alpha$ ) promoter regulates expression of an angiostatin-endostatin fusion gene [32]. To form the transposon, the transcriptional cassettes are flanked by Sleeping Beauty T2 transposon inverted terminal repeats [33].

Murine CT26 colon carcinoma is a transplantable tumor that shows strong hypervascularization and is an effective model for evaluation of an angiostatic, antitumor approach. Balb/c mice were injected with CT26 colorectal tumor cells intrasplenically, subsequently allowing the cells to seed into the liver and initiate localized tumor growth. Two days following tumor seeding, animals were hydrodynamically injected (see Materials and Methods) with $10 \mu \mathrm{g}$ (low dose) or $25 \mu \mathrm{g}$ (high dose) of pKT2/BidAEL along with either $0,5,10,12.5$ or $25 \mu \mathrm{g}$ of SB transposase plasmid. Plasmid delivery to the liver was assessed by in vivo bioluminescence imaging of luciferase expression at 24 hours, 1 week and two weeks post-injection. Figure 1B depicts a representative animal with localized hepatic expression of luciferase imaged at 24 hours after plasmid injection. Levels of expression in the high dose and low dose transposon groups are depicted in Figures 2A and 2B respectively. All animals, regardless of presence or absence of $\mathrm{SB}$ plasmid, showed similar levels of expression, with the highest levels observed at 24 hours and 1 week postinjection, ranging from $6 \times 10^{9}$ to $5 \times 10^{10}$ photons $/ \mathrm{sec}$. 


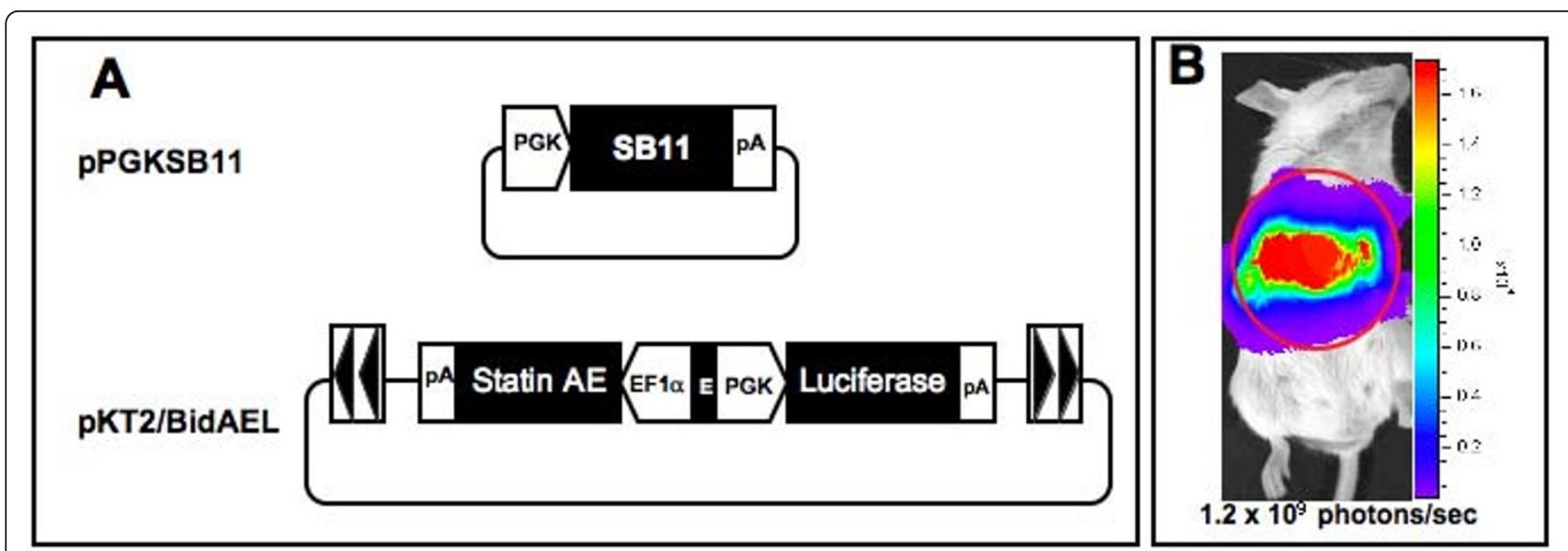

Figure 1 Gene delivery and expression of firefly luciferase in the liver. A, Plasmid constructs used in gene delivery to the liver. PGK, phosphoglycerate kinase; PA, SV40 polyadenylation signal; SB11, Sleeping Beauty 11 transposase gene; EF1- $\alpha$, elongation factor1- $\alpha$; E, CpG-free synthetic enhancer; inverted arrowheads, T2 transposon inverted repeats. B, Representative mouse from the group that received $10 \mu \mathrm{g}$ transposon and $10 \mu \mathrm{g} \mathrm{SB}$ plasmids showing gene delivery and expression in the liver 24 hrs following hydrodynamic gene delivery.

At 2 weeks post-injection, luciferase expression was markedly reduced to levels that were approximately 1000 -fold lower (mean of $1.2 \times 10^{7}$ photons/sec). Levels of luciferase expression in both the high and low dose transposon groups were similar, and both groups exhibited a similar time course of expression over two weeks. These results confirm hepatic delivery and subsequent transgene expression of the therapeutic pKT2/BidAEL plasmid.

Effect of antiangiogenic transposon gene therapy on tumor load

Animals were sacrificed 21 days after tumor infusion, and the effect of antiangiogenic transposon treatment on tumor burden was gauged by total liver weight (Figures 3A and $3 B$ ) as well as reduction of the number of tumor metastases (Figures $4 \mathrm{~A}$ and $4 \mathrm{~B}$ ). The mean liver weight of untreated tumor bearing animals $(4.2+/-2.1 \mathrm{~g})$ was significantly higher $(\mathrm{P}<0.01)$ than that of each of the groups of antiangiogenic transposon-treated tumor bearing animals, in which the mean weights ranged from 1.37 to $1.65 \mathrm{~g}$, as well as the non-tumor bearing control group, which had a mean liver weight of $0.9 \mathrm{~g}$. There was no significant difference in liver weights between groups that were co-injected with either $0,12.5$, or $25 \mu \mathrm{g}$ of SB transposase plasmid along with $25 \mu \mathrm{g}$ of Statin AE transposon or between any of these groups and the non-tumor bearing control group (Figure 3A).
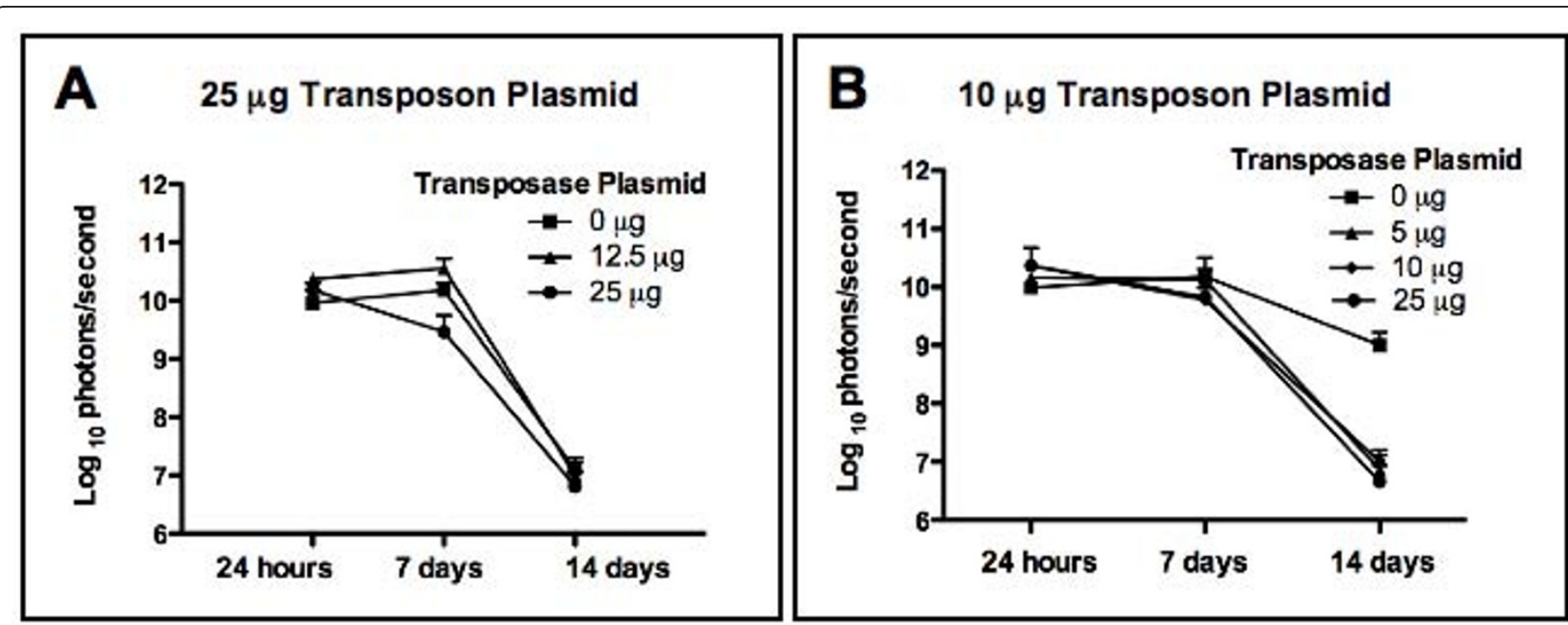

Figure 2 Time course and levels of luciferase expression following hydrodynamic gene delivery. Animals were injected with plasmids as indicated below, and imaged at various time points for in vivo luciferase gene expression (mean +/- positive SD). A, $25 \mu \mathrm{g}$ high dose transposon plasmid; $\mathbf{B}, 10 \mu \mathrm{g}$ low dose transposon plasmid. 


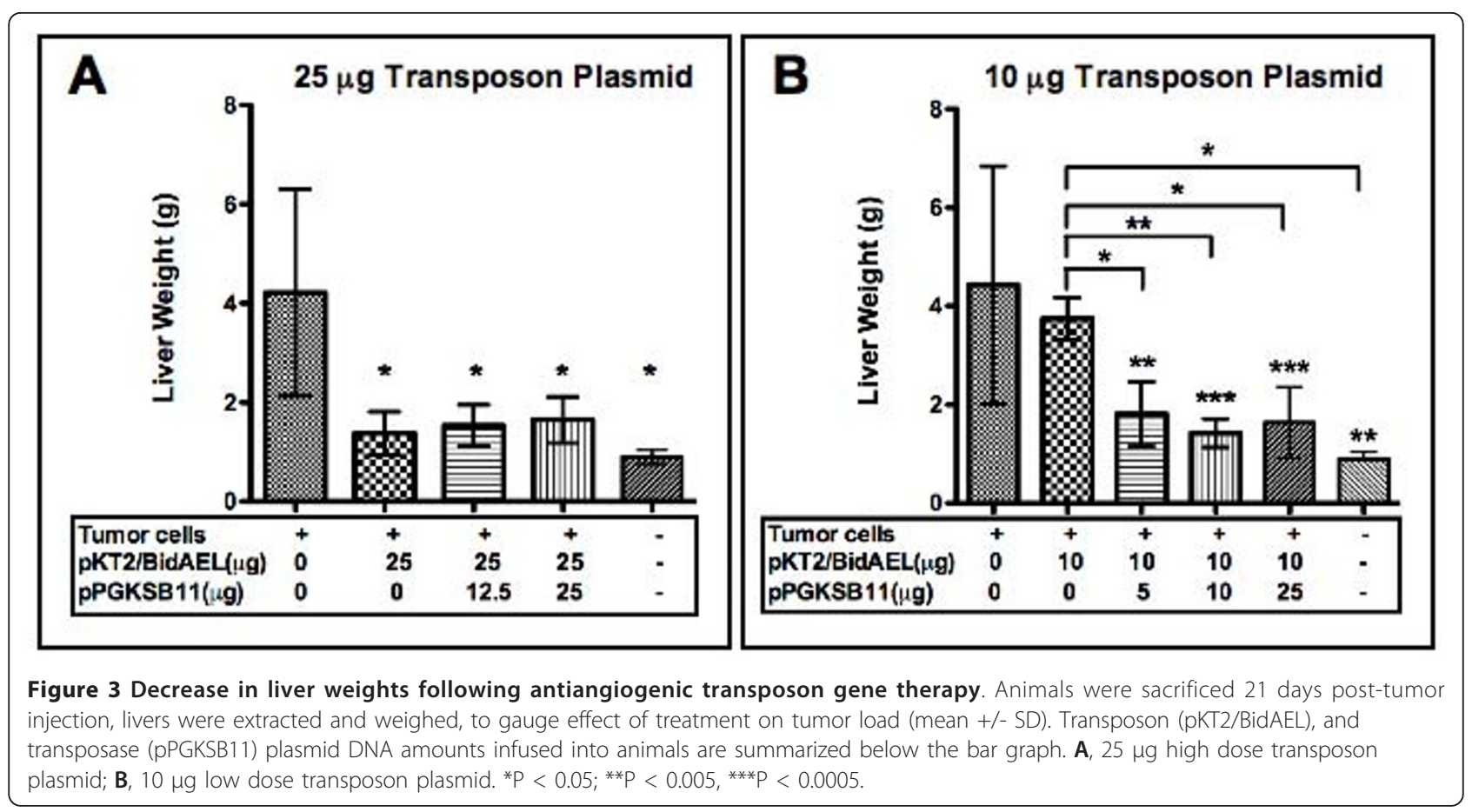

Tumor-bearing animals that were injected with low dose $(10 \mu \mathrm{g})$ Statin AE transposon plasmid alone had a mean liver weight of $3.75+/-0.43 \mathrm{~g}$, which was not significantly lower than that of the untreated tumor-bearing control group $(4.4+/-2.4 \mathrm{~g})$. However, animals that were co-injected with either 5,10 , or $25 \mu \mathrm{g}$ of SB transposase plasmid had mean liver weights ranging from $1.4+/$ $0.3 \mathrm{~g}$ to $1.8+/-0.7 \mathrm{~g}$ and these were significantly lower
$(\mathrm{P}<0.0005)$ than untreated tumor-bearing control animals and tumor-bearing animals administered $10 \mu \mathrm{g}$ transposon plasmid alone (Figure 3B).

In all groups of animals treated with a high dose $(25 \mu \mathrm{g})$ of Statin AE transposon DNA, there was a significant reduction in the number of liver tumor nodules in comparison with that of untreated tumor bearing animals $(\mathrm{P}<0.05)$, whether or not SB transposase plasmid

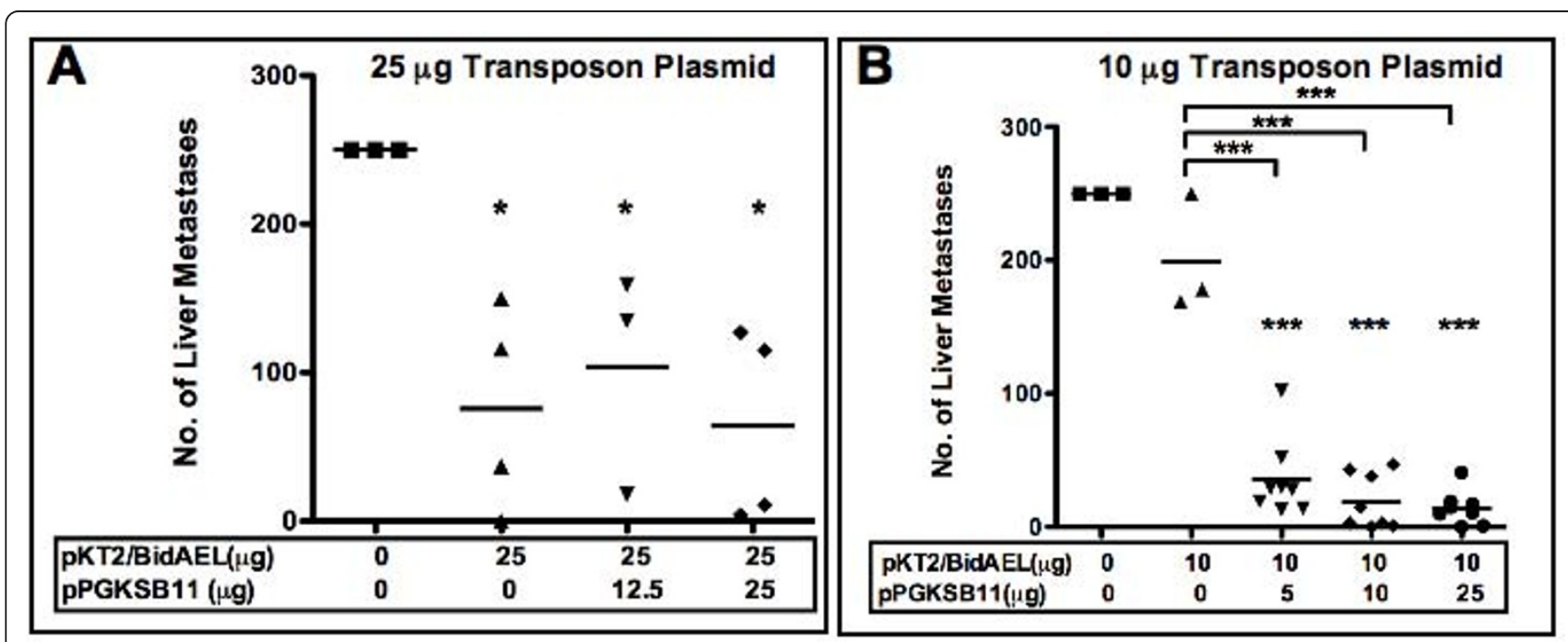

Figure 4 Effects of antiangiogenic transposon gene therapy on liver metastases. Livers were extracted from sacrificed animals and tumor nodules on the liver were enumerated. Transposon (pKT2/BidAEL), and transposase (pPGKSB11) plasmid DNA amounts infused into animals are summarized below the graph. A, $25 \mu \mathrm{g}$ high dose transposon plasmid (bar indicates mean of $\mathrm{n}=3-4$ ); $\mathbf{B}, 10 \mu \mathrm{g}$ low dose transposon plasmid, (bar indicates mean of $n=8$ ). ${ }^{*} P<0.05$; ${ }^{* *} P<0.0005$. 
was co-injected (Figure 4A). In addition, the results were similar regardless of dosage of SB transposase.(12.5 or 25 $\mu \mathrm{g})$. However, in animals treated with a low dose $(10 \mu \mathrm{g})$ of Statin AE transposon alone (Figure 4B), antitumor effectiveness was observed only in animals co-administered SB transposase-encoding DNA. Infusion of $10 \mu \mathrm{g}$ Statin AE transposon alone caused only a minimal (nonsignificant) reduction in liver tumor nodules compared to untreated tumor-bearing animals. In contrast, there was a significant decrease in metastases $(\mathrm{P}<0.0001)$ observed in animals co-infused with $5 \mu \mathrm{g}(36+/-29), 10$ $\mu \mathrm{g}(19+/-20)$, or $25 \mu \mathrm{g}(14+/-12) \mathrm{SB}$ plasmid, i.e a trend toward decreased nodule formation with increasing doses of SB transposase plasmid.

\section{Effect of antiangiogenic transposon treatment on histological indices of metastatic tumors}

To evaluate the effect of anti-angiogenic gene therapy, animals were sacrificed at 3 weeks post-injection and livers were analyzed histologically for the presence and extent of replacement of normal liver by tumor tissue. Livers of untreated tumor bearing mice were enlarged and contained multiple, coalescing pale nodules that replaced nearly all of the normal hepatic parenchyma (Figure 5A), while the livers of animals treated with Statin AE transposon plasmid were smaller in size and contained tumor nodules that replaced a much lower volume of tissue (Figure 5B).

The hepatic replacement area (HRA) (defined as the percentage of normal hepatic tissue that has been displaced by metastatic tumor) was assessed morphometrically in histological sections for the low dose transposon mice, and is summarized in Figure $5 \mathrm{C}$. In untreated tumor bearing controls, the hepatic replacement area was close to $100 \%$ in all 3 mice examined (i.e. there was essentially no normal liver tissue detectable in these animals). Animals treated with low dose transposon alone had a mean HRA of $68.3 \%$, and animals that received various doses of SB plasmid had mean HRAs ranging from 12.83 to $35.26 \%$. The group that received $10 \mu \mathrm{g}$ of SB transposase plasmid was the only group that differed significantly from untreated controls. Despite the striking means, the lack of significant difference observed for the other treated groups was probably due to low sample size and variability among samples.

\section{Effect of antiangiogenic transposon gene therapy on tumor endothelium and proliferation of colorectal cancer cells}

Tumors were evaluated for the effect of low dose Statin AE transposon treatment on proliferation of tumor cells and on tumor vascularization by histomorphometric evaluation of serial immunohistochemistry sections using antibodies directed against Ki67 and CD31, respectively
(Figure 6A, B, and 6C). The highest level of tumor cell proliferation (9.6\%) was observed in the tumor bearing untreated group, with significantly lower mean proliferation indices (2.6-4.8\%) seen in animals that received Statin AE transposon plasmid with or without SB transposase plasmid (Figure 6B). Similarly, tumor tissues from tumor bearing untreated mice had the highest index of CD31 positive blood vessels (9\%). In comparison, animals that were treated with Statin AE transposon plasmid with or without SB transposase plasmid demonstrated significantly lower mean (3-3.5\%) of vessel density (Figure 6C). There was no significant difference between animals treated with vs. without SB transposase plasmid for either Ki67 or CD31 preparations.

\section{Extended survival of tumor-bearing mice treated with antiangiogenic transposon}

An additional cohort of tumor bearing animals was tested to determine the effect of SB transposase plus statin AE transposon administration on survival. Animals were implanted with $5 \times 10^{4}$ tumor cells intrasplenically as described in Materials and Methods and then 3 days later, injected hydrodynamically with $10 \mu \mathrm{g}$ SAE transposon with or without $25 \mu \mathrm{g}$ SB11 transposase plasmid. The animals were imaged weekly, demonstrating high level luciferase expression and verifying effective hydrodynamic gene delivery (data not shown). All untreated animals, as well as animals treated with transposon alone, succumbed to tumor and were euthanized by day 22 and 23 respectively (Figure 7 ). In contrast, survival was significantly extended ( $p<0.0001)$, to day 40 in some cases, for animals that were treated with both transposon and transposase encoding plasmids. Tumor burden was evaluated at time of sacrifice, verifying the growth of hepatic metastases (not shown). We conclude that inhibition of CT26 tumor growth brought about by coinfusion of SAE transposon plus SB transposase encoding plasmid confers increased survival in tumorbearing animals.

\section{Discussion}

StatinAE gene transfer using a Sleeping Beauty transposon vector resulted in antitumoral effects in an animal model of colorectal cancer metastatic to the liver. Significant tumor growth inhibition was seen in mice injected with a high (25 $\mu \mathrm{g})$ dose of antiangiogenic transposon plasmid, in which co-injection of the SB transposase did not achieve any further tumor regression. In contrast, antitumor activity of the Statin AE transposon administered at a lower dose $(10 \mu \mathrm{g})$ was dependent on co-infusion of SB transposaseencoding plasmid. These results suggest that antiangiogenic gene therapy using the SB non-viral transposon system has the potential to be an effective treatment for colorectal cancer metastatic to the liver, and that this process is 


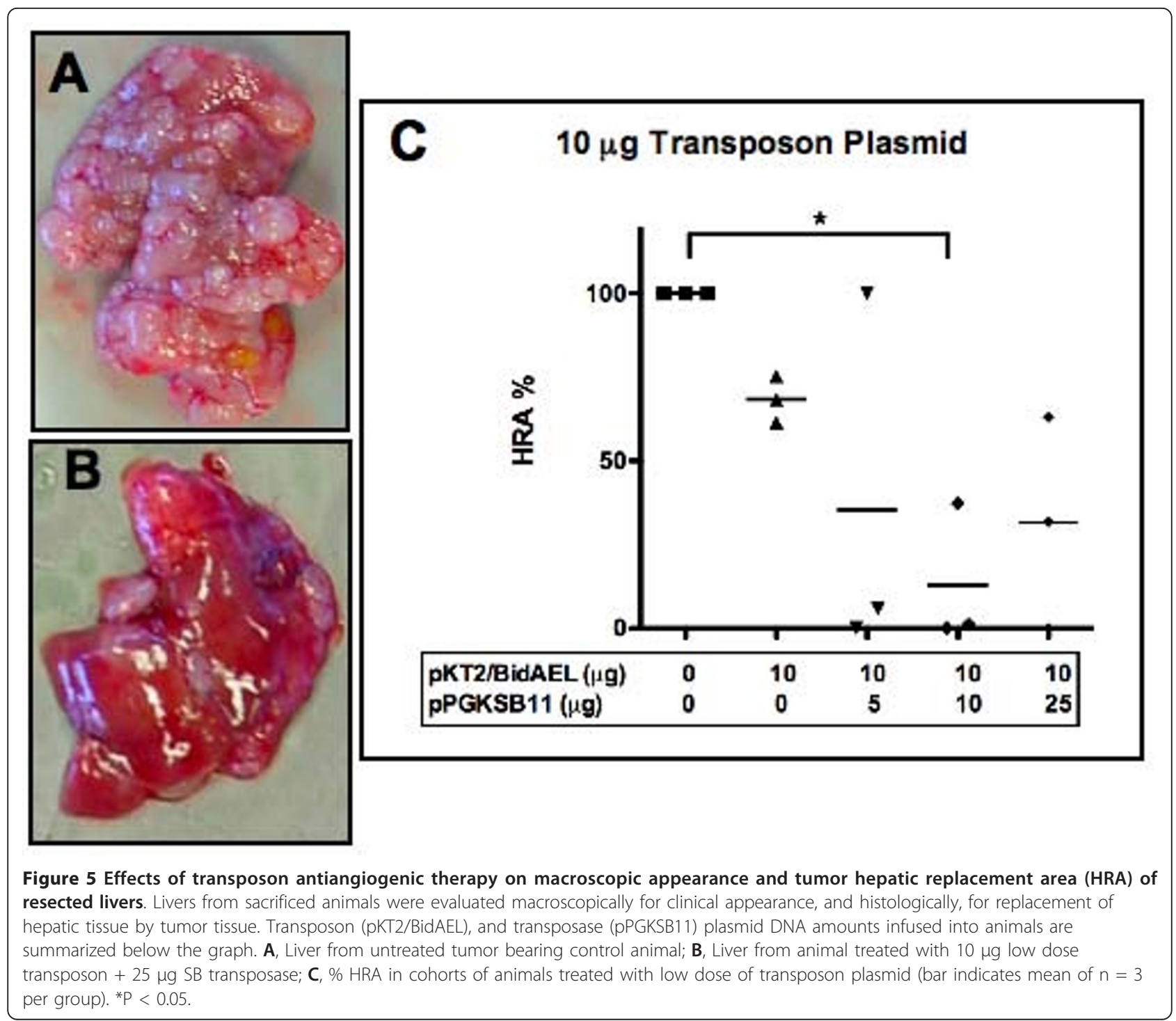

dependant on the transposition process under transposon dose-limiting conditions.

Although antiangiogenic proteins show great promise in preclinical cancer models, their effectiveness in the clinic, especially when administered alone, has been limited [34-36]. The effect of VEGF on tumor and vascularization has been studied by many groups, demonstrating it's pivotal role in increasing vascular permeability, tumor growth and metastasis $[37,38]$. In pre-clinical studies, VEGF targeting strategies have demonstrated significant antivascular effects and tumor growth inhibition. Although several drugs and small molecule inhibitors of VEGF are being clinically tested, their efficacy as monotherapeutic agents in advanced stage disease has been discouraging, due to short half-lives and dose limiting toxicities [39]. However, there is a significant survival benefit when VEGF-targeted therapy is combined with standard chemotherapy for metastatic colorectal cancer [36]. Also, most inhibitors of angiogenesis exert their effect against newly formed blood vessels rather than existing vasculature. The inability of these agents to completely eradicate disease presents a serious challenge for patients with advanced malignant tumors. For most angiogenesis inhibitors, tumor growth resumes after cessation of therapy [39]. This necessitates administration of high doses and prolonged treatment with antiangiogenic proteins to obtain a sustained therapeutic effect.

Delivery of gene sequences encoding recombinant inhibitors of angiogenesis potentially allows for localized and sustained expression, which could reduce the risk of systemic toxicity and perhaps increase efficacy. Other advantages include the possibility of expressing multiple antiangiogenic gene products that act via different mechanisms, thereby increasing the effectiveness of the 


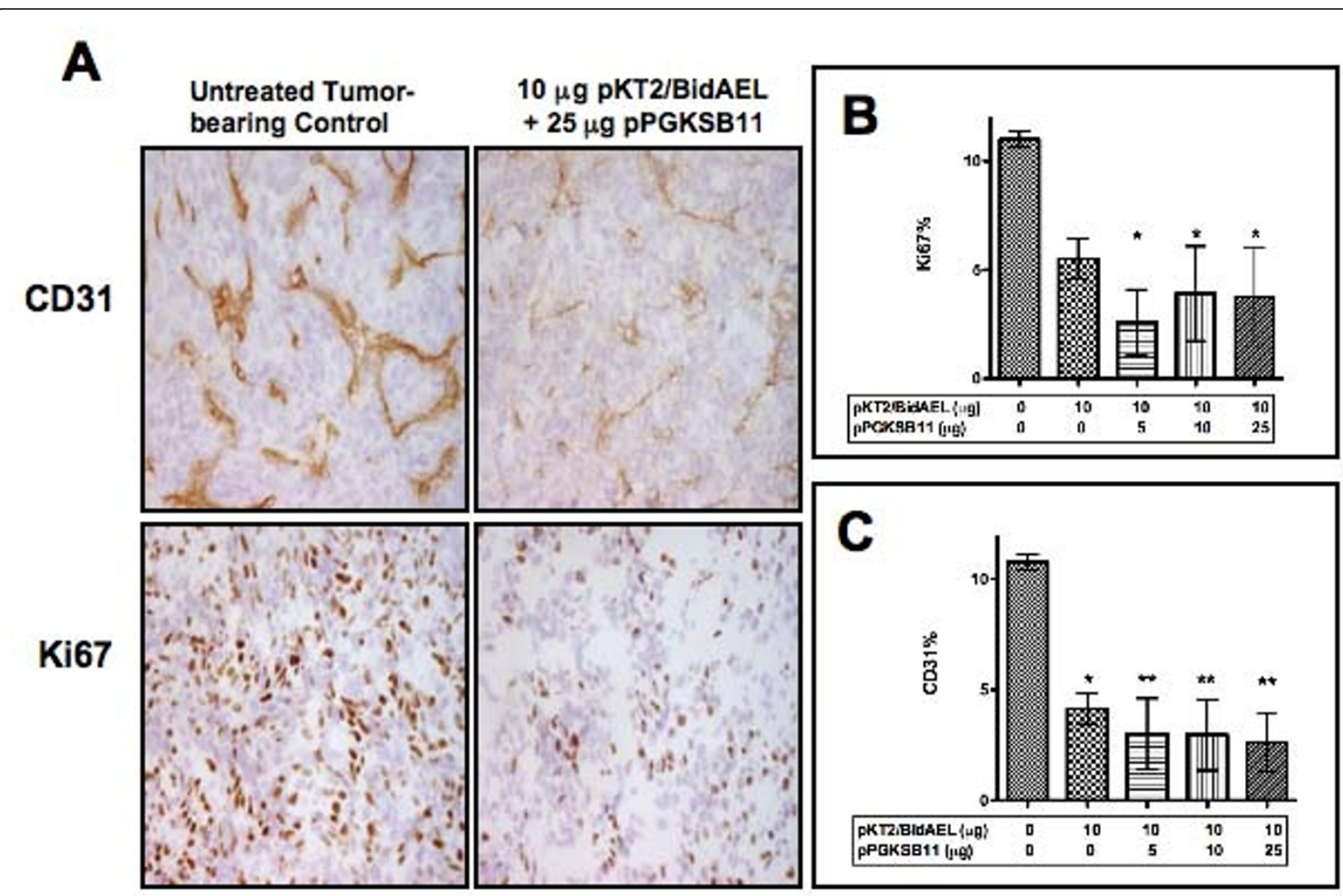

Figure $\mathbf{6}$ Immunohistochemical analysis of tumor tissues for cell proliferation and tumor vasculature. A, $I H C$ staining to determine tumor vasculature and proliferation was performed in $5 \mu \mathrm{m}$ sections on liver. Samples from animals treated with $10 \boldsymbol{\mu g}$ transposon plasmid and varying amounts of transposase plasmids were stained using anti-CD31 (tumor vasculature) and anti-Ki67 antibodies (tumor proliferation) and visualized by light microscopy. B, Percent positively staining area using Ki67 antibody; C, Percent positively staining area using CD31 antibody. Percentage of positively stained areas in respective groups was determined by measuring the area of immunopositivity vs. total tissue area in each of 10 random fields (mean +/- SD). Transposon (pKT2/BidAEL), and transposase (pPGKSB11) plasmid DNA amounts infused into animals are summarized below the graph. ${ }^{*} \mathrm{P}<0.05 ;{ }^{* *} \mathrm{P}<0.005$.

therapy. The antitumor effects of introducing gene sequences encoding endogenous anti-angiogenic proteins endostatin and angiostatin have been characterized in several studies, and their synergistic effects when used in combination (either in individual or separate vectors) is well documented [29-31,40]. We therefore investigated an angiostatin-endostatin fusion protein previously shown to confer potent antitumor effects in a subcutaneous melanoma model in vivo [32].

The integrating Sleeping Beauty (SB) transposon system circumvents the primary limitation of non-viral plasmid based gene transfer, i.e. the transient duration of gene expression that fails to give way to long-term expression. SB-mediated transposition has been shown to occur in a variety of cultured cell types, in zebrafish [41] and mouse embryos and germ cells [42], in human primary blood lymphocytes [26], and in mouse somatic tissues, including the lung and the liver [25,43-46]. SB-mediated transposition in mouse liver has been verified in several laboratories by recovery and sequencing of transposon-chromosome junction sequences [22,25,44,47-52].

SB has also been used successfully to deliver antitumor genes to neoplastic tissue. Ohlfest et al successfully used the SB transposon system to deliver a cocktail of antiangiogenic genes to human glioblastoma xenografts in mice, subsequently observing increased survival and sustained regression of tumor [27]. Wu et al compared the efficacy of interferon-gamma immunogene therapy using non-integrating plasmid vectors vs SB plasmid vectors in a syngeneic glioma model. Only animals co-injected with SB transposase plasmid exhibited prolonged expression of interferon-gamma and a significant increase in survival (3 weeks), while expression in animals treated with transposon plasmid alone was undetectable after 1 week [53].

While hydrodynamic tail vein injection has been used very effectively for delivering DNA to liver tissue in rodents, extending this technique to humans is still 


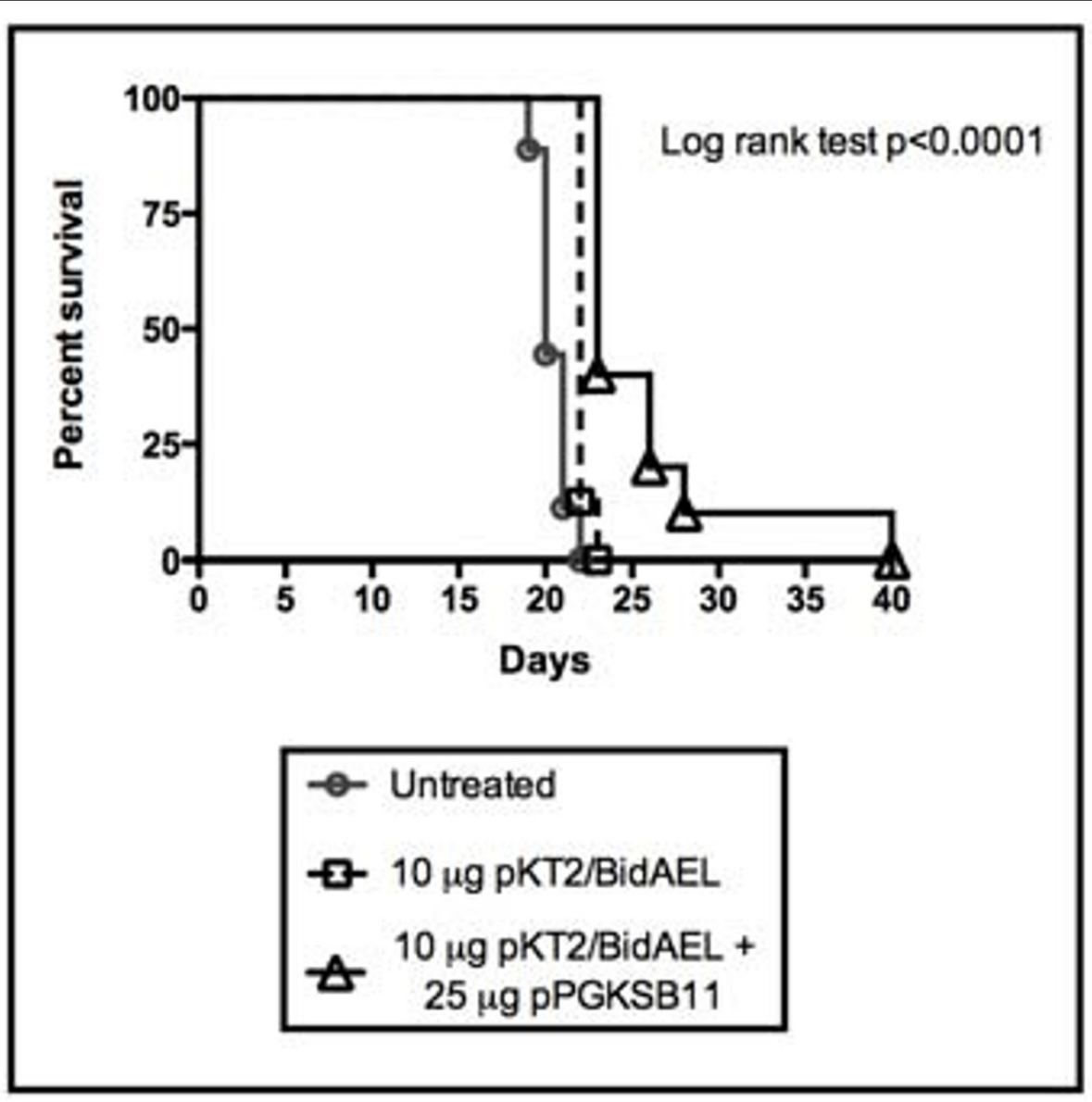

Figure 7 Survival of mice treated with antiangiogenic transposon. The Kaplan-Meier plot shows the fraction of animals surviving vs. time after implantation of CT26 cells at day 0 with subsequent hydrodynamic injection of SAE transposon with or without SB transposase-encoding plasmid on day 3 (8 mice per group). Differences in survival between groups was evaluated by log-rank analysis. ${ }^{*} \mathrm{P}<0.005$.

implausible. However, several laboratories have reported the use of balloon occlusion catheters for successful gene delivery to the liver in large animals. Delivery of DNA into occluded rabbit liver under $\mathrm{X}$-ray guidance [54], into the left lateral lobe of pig liver by catheterization and occlusion of the portal vein [55], and to the whole liver of pig and dog via the inferior vena cava (IVC) with double balloon occlusion above and below the IVC-hepatic vein conjunctions [56,57] have been reported. Liu et al have developed a device that uses high pressure from a gas cylinder and a computercontrolled switch to drive and regulate DNA injection in pigs [58]. Use of this device, combined with vessel occlusion and image guided catheterization to achieve site specificity, was found to provide effective gene delivery [59]. Overall, these results from large animal studies demonstrate that modifications of the hydrodynamic technique can potentially be applied to humans.
Our results demonstrate that animals injected with both transposon and transposase-encoding plasmids survive significantly longer than untreated control animals, or animals treated with transposon plasmid alone. However, transgene expression declines over time, leading to eventual emergence of tumor metastases. Transgene-specific immune responses, both humoral and cell-mediated, have been described. Aronovich et al have shown that prolonged expression of $ß$-glucuronidase after SB transposonmediated delivery elicited an immune response against transgene-expressing cells, which were subsequently eliminated (22). Lutzko et al have characterized humoral immune responses against transgene products by ELISA, and have demonstrated cellular immune responses using lymphocyte proliferation assays [60]. Relative persistence of transgene expression is also mouse-strain dependant. Injection of recombinant adenovirus expressing human alpha-1-antitrypsin (hAAT), resulted in persistent, circulating levels of hAAT in $\mathrm{C} 57 \mathrm{Bl} / 6$ mice, while Balb/c mice 
rapidly neutralized the transgene product [61]. Hodges et al have shown that hydrodynamic injection of $\mathrm{CpG}$ replete, supercoiled human Factor IX encoding plasmid in $\mathrm{Balb} / \mathrm{c}$ mice resulted in loss of transgene expression after 3-4 weeks, while therapeutic levels of Factor IX expression were maintained in mice that received $\mathrm{CpG}$ depleted plasmid [62]. Any or all of these factors could account for the decline in gene expression observed in our study. Modulation or counteraction of the immune response is therefore essential in maintaining sustained transgene expression and continued suppression of tumor metastases.

Here, we have shown that the SB transposon system can be used to successfully deliver antiangiogenic genes and inhibit colorectal tumors metastatic to the liver. We observed that treatment with a high dose of antiangiogenic transposon plasmid appeared to be effective with no requirement for co-delivery of SB transposase. However, antitumor effectiveness of Statin-AE transposon when administered at low dose $(10 \mu \mathrm{g})$ was dependent on SB transposase. Since transposon delivery in large animals and in humans is likely to be infrequent, the low dose treatment is considered to be more representative of the low level of gene transfer that is to be expected in a clinical setting. In this case, co-delivery of the SB transposase encoding plasmid is required, thereby enabling sustained expression of the therapeutic antiangiogenic transgene through transposition, and thus continued inhibition of tumor growth.

\section{Conclusions}

Patients who would benefit the most from antiangiogenic gene therapy may be those with early metastatic disease or even preoperative or perioperative disease [10,11]. In this study, we have shown significant inhibition of liver metastases using the SB transposon system. However, complete eradication of tumor was not seen in any of the treated groups. Clinically, antiangiogenic gene therapy may be most well suited for use as an adjuvant therapy when administered along with existing treatment options of surgery and/or chemotherapy [8,9]. This combination therapy may facilitate the use of low dose chemotherapy in patients. Future preclinical studies will evaluate transposition and animal survival in combination therapy of SB with other cytoreductive treatments.

\section{Methods}

\section{Plasmids and cell lines}

The murine CT26 colon carcinoma cell line (ATCC, Manassas, VA) was routinely maintained in RPMI 1640 medium supplemented with $10 \%$ fetal bovine serum and $1 \%$ penicillin/streptomycin/fungizone. SB transposon and transposase containing plasmids were constructed using standard molecular cloning techniques. The transposon plasmid containing the bidirectional promoter
(pKT2/LuBiG) is described in Multhaup et al [63]. Statin AE was cloned as an EcoRI-NotI fragment from pT2/ CAE [27] into plasmid pKT2/PGCL to generate plasmid pKT2/BidAEL. The SB transposase plasmid pPGK-SB11 consists of the phosphoglycerate kinase promoter regulating transcription of the SB11 transposase as described previously [49].

\section{Establishment of hepatic tumors}

Female Balb/c mice (6-8 weeks of age) were obtained from $\mathrm{NIH}$ (Frederick, MD) and maintained under specific pathogen-free conditions. All animals were treated according to the NIH Guidelines for Animal Care with approval of the IACUC of the University of Minnesota. Exponentially growing CT26 colon carcinoma cells were harvested by trypsinization, suspended in Hanks' balanced salt solution, and $1 \times 10^{5}$ viable cells (as determined by trypan blue exclusion) in a total volume of $200 \mu$ l were injected intraspenically as previously described [64]. Briefly, animals were administered $0.2 \mathrm{ml}$ of an anaesthetization cocktail consisting of ketamine $\mathrm{HCl}(8 \mathrm{mg} / \mathrm{ml}$; Phoenix Scientific, St. Joseph, MO), acepromazine maleate $(0.1 \mathrm{mg} /$ $\mathrm{ml}$; Phoenix Scientific), and butorphanol tartarate $(0.01 \mathrm{mg} / \mathrm{ml}$; Fort Dodge Animal Health, Overland Park, $\mathrm{KS})$. A peritoneal incision was made to expose the spleen. Cells were delivered directly to the spleens of recipient animals, and a sterile cotton tip applicator was used to apply pressure over the injection site for 3 minutes. A splenectomy was subsequently performed to remove the primary site of tumor inoculation, thus confining tumor formation to the liver. The incision was closed with staples and the animal allowed to recover.

\section{Hydrodynamic delivery of transposon and transposase encoding plasmids}

3 days following tumor cell implantation, 8 animals per experimental group were weighed and administered $0.03 \mathrm{ml}$ of an anaesthetization cocktail as described above. Lactated Ringers solution was added to plasmid DNA to bring the total volume (in $\mathrm{mL}$ ) equivalent to $10 \%$ of the total body weight (in grams) for each mouse. The plasmid DNA solution was then injected rapidly through the tail vein of the animal in a period of 4-8 seconds. Animals that did not receive the injection in less than 8 seconds were omitted from the study. Injected animals were placed on a heating pad and monitored until recovery from anesthesia [65].

\section{In vivo luciferase imaging}

Animals were anaesthetized using a sedative dose (400 $\mu$ l i.p. for a 25 gram mouse) of the anaesthetization cocktail described above, followed by intraperitoneal injection of $100 \mu \mathrm{l}$ of luciferin substrate $(28.5 \mathrm{mg} / \mathrm{ml}$; Caliper Life Sciences, Hopkington, MA). Five minutes 
following luciferin injection, mice were imaged for 1 second, using an intensified CCD camera (Xenogen Corporation, Alameda, CA). Raw values were recorded as photons of light emitted per second [66].

\section{Animal necropsy and immunohistochemistry}

Animals were sacrificed 21 days post-tumor seeding, or at the time of declining health in the survival study. Animals were euthanized according to the University of Minnesota IACUC-approved protocols. The date of euthanasia was recorded as the end day of survival and autopsy was subsequently performed. Liver weights and tumor nodule counts were recorded. Intact livers from all groups except the nontumor bearing control group were snap frozen in 2-methylbutane and stored in liquid nitrogen. Frozen tissue blocks were sectioned at 5 microns, mounted on slides and stored at $-80^{\circ} \mathrm{C}$ until use, at which time they were allowed to come to room temperature, fixed in acetone for $10 \mathrm{~min}$, and either stained routinely with hematoxylin and eosin or immunostained using antibodies directed against CD-31 or Ki67. For this procedure, endogenous peroxidase activity was blocked with $0.3 \% \mathrm{H}_{2} \mathrm{O}_{2}$ for 15 minutes. Undiluted avidin and biotin were applied for 15 minutes each to block nonspecific biotin (DAKO X0590). In addition a protein serum block was applied for 15 minutes. The appropriate primary antibody (rat anti-mouse CD 31; BD Pharmingen Cat \#550274 or rat anti-mouse Ki-67; Dako Cat \# M7249) was applied at a dilution of 1:100 (CD31) or 1:25 (Ki-67) for 60 minutes. The secondary antibody (biotinylated anti-rat 1:300; Vector Laboratories Cat \# BA-4001) was applied for 30 minutes followed by undiluted streptavidin-HRP (Dako Cat \# K1016). The brown reaction product was detected using 3,3'diamino-benzidine (Dako Cat \# K3466). Sections were counterstained with Mayer's hematoxylin. For negative control sections, normal rat serum was substituted for the primary antibody.

\section{Histomorphometry}

The H\&E stained tissue sections were imaged using a $1 \times$ objective and the immunohistochemical sections were imaged using a $20 \times$ objective. The $1 \times$ images were captured with a Nikon E800 microscope and a Nikon DXM 1200 digital camera (Nikon Instruments Inc., Tokyo, Japan) and included the entire area of tissue. The 20× images were captured with an Olympus BX40 (Olympus, USA) and a SPOT Insight digital camera (Diagnostic Instruments Inc., Sterling Heights, IL) and were taken only within tumor tissue. Measurement of images (1X) of the H\&E stained sections were used for morphometric evaluation of \% tumor area using ImagePro Plus 6.2 (Media Cybernetics, Silver Springs,
Maryland). Tumor area (\%) was determined by tracing the outer margin of each metastatic lesion and determining its area, summing the areas of all metastatic lesions in the tissue section, and dividing this sum by the total area of the tissue section.

Images of the immunohistochemistry sections were analyzed using Image-Pro Plus 6.2 (Media Cybernetics, Silver Springs, Maryland). Areas within the tumors that stained positively for Ki-67 or CD31 by IHC were differentiated from negative areas by using the threshold command. 5 randomly selected fields (field size approximately 265,350 square microns) from tumor tissue in each section were evaluated. The total area of tissue as well as the area of immunopositivity for CD-31 or Ki-67, respectively, were measured $\left(\mu \mathrm{m}^{2}\right)$. The area of immunopositivity was then recorded as a percentage of the total area. Results from the 5 fields were then averaged for each section.

\section{Statistical analysis}

Data were analyzed using GraphPad Prizm 5.0 software (GraphPad Software Inc., San Diego, CA). Statistical analysis of differences between groups was determined using one way analysis of variance (ANOVA), with Tukey's post test analysis. Animal survival was evaluated by the Kaplan-Meier product limit method, comparing differences among animal groups by the log rank test. Differences were considered significant when $\mathrm{P}<0.05$ for ANOVA.

\section{Acknowledgements}

This project was supported by grant CA120383 from the National Cancer Institute. BSS is supported by grant F30DE020210 from the National Institute of Dental and Craniofacial Research.

\section{Author details}

${ }^{1}$ Center for Genome Engineering, University of Minnesota, Minneapolis, MN 55455, USA. ${ }^{2}$ Gene Therapy Program, Institute of Human Genetics, University of Minnesota, Minneapolis, MN 55455, USA. ${ }^{3}$ Department of Genetics, Cell Biology and Development, University of Minnesota, Minneapolis, MN 55455, USA. ${ }^{4}$ Department of Veterinary Population Medicine, University of Minnesota, St. Paul, MN 55108, USA. ${ }^{5}$ Department of Surgery, University of Minnesota, MN 55455, USA. ${ }^{6}$ Department of Pharmacology, University of Minnesota, Minneapolis, MN 55455, USA.

\section{Authors' contributions}

LRB carried out the molecular cloning, experimental design, data acquisition/ analysis, and drafted the manuscript. KPP carried out the plasmid hydrodynamic injections, and for animal care. BS performed surgical implantation of tumor cells. AH participated in animal experiments and data collection. JP and CSS carried out immunohistochemical assays and quantitation. DS and SR provided key experimental resources and critically reviewed the manuscript. RSM conceived the study, participated in its experimental design and co-wrote the manuscript. All authors read and approved the final manuscript.

\section{Competing interests}

R. Scott Mclvor is a founder and manager of Discovery Genomics, Inc.

Received: 19 April 2010 Accepted: 10 February 2011

Published: 10 February 2011 


\section{References}

1. Edwards BK, Ward E, Kohler BA, et al: Annual report to the nation on the status of cancer, 1975-2006, featuring colorectal cancer trends and impact of interventions (risk factors, screening, and treatment) to reduce future rates. Cancer 2009, 1:544-73.

2. Mayo SC, Pawlik TM: Current management of colorectal hepatic metastasis. Expert Rev Gastroenterol Hepatol 2009, 3:131-44.

3. Carpizo DR, D'Angelica M: Liver resection for metastatic colorectal cancer in the presence of extrahepatic disease. Lancet Oncol 2009, 10:801-9.

4. de Jong MC, Pulitano C, Ribero D, et al: Rates and patterns of recurrence following curative intent surgery for colorectal liver metastasis: An international multi-institutional analysis of 1669 patients. Ann Surg 2009, 250:440-8.

5. Takebayashi Y, Aklyama S, Yamada K, Akiba S, Aikou T: Angiogenesis as an unfavorable prognostic factor in human colorectal carcinoma. Cancer 1996, 78:226-31.

6. Vermeulen $P B$, Van den Eynden $G G$, Huget $P$, et al: Prospective study of intratumoral microvessel density, p53 expression and survival in colorectal cancer. Br J Cancer 1999, 79:316-22.

7. Ivy SP, Wick JY, Kaufman BM: An overview of small-molecule inhibitors of VEGFR signaling. Nat Rev Clin Oncol 2009, 6:569-79.

8. Segal NH, Saltz LB: Evolving treatment of advanced colon cancer. Annu Rev Med 2009, 60:207-19.

9. Sobrero A, Ackland S, Clarke S, et al: Phase IV study of bevacizumab in combination with infusional fluorouracil, leucovorin and irinotecan (FOLFIRI) in first-line metastatic colorectal cancer. Oncology 2009, 77:113-9.

10. Benjamin LE, Golijanin D, Itin A, Pode D, Keshet E: Selective ablation of immature blood vessels in established human tumors follows vascular endothelial growth factor withdrawal. J Clin Invest 1999, 103:159-65.

11. Folkman J: Antiangiogenic gene therapy. Proc Natl Acad Sci USA 1998, 95:9064-6

12. Kong $H \mathrm{~L}$, Hecht $\mathrm{D}$, Song $\mathrm{W}$, et al: Regional suppression of tumor growth by in vivo transfer of a cDNA encoding a secreted form of the extracellular domain of the flt-1 vascular endothelial growth factor receptor. Hum Gene Ther 1998, 9:823-33.

13. Mahasreshti PJ, Kataram M, Wang MH, et al: Intravenous delivery of adenovirus-mediated soluble FLT-1 results in liver toxicity. Clin Cancer Res 2003, 9:2701-10.

14. Chen $C T$, Lin J, Li Q, et al: Antiangiogenic gene therapy for cancer via systemic administration of adenoviral vectors expressing secretable endostatin. Hum Gene Ther 2000, 11:1983-96.

15. Feldman AL, Restifo NP, Alexander HR, et al: Antiangiogenic gene therapy of cancer utilizing a recombinant adenovirus to elevate systemic endostatin levels in mice. Cancer Res 2000, 60:1503-6.

16. Shi W, Teschendorf C, Muzyczka N, Siemann DW: Adeno-associated virusmediated gene transfer of endostatin inhibits angiogenesis and tumor growth in vivo. Cancer Gene Ther 2002, 9:513-21.

17. Gorrin-Rivas MJ, Arii S, Mori A, Kaneda Y, Imamura M: Mouse macrophage metalloelastase gene delivery by HVJ-cationic liposomes in experimenta antiangiogenic gene therapy for murine CT-26 colon cancer. Int J Cancer 2001, 93:731-5

18. Uesato M, Gunji $Y$, Tomonaga $T$, et al: Synergistic antitumor effect of antiangiogenic factor genes on colon 26 produced by low-voltage electroporation. Cancer Gene Ther 2004, 11:625-32

19. Wen J, Matsumoto K, Taniura N, Tomioka D, Nakamura T: Inhibition of colon cancer growth and metastasis by NK4 gene repetitive delivery in mice. Biochem Biophys Res Commun 2007, 358:117-23.

20. Wen J, Matsumoto K, Taniura N, Tomioka D, Nakamura T: Hepatic gene expression of NK4, an HGF-antagonist/angiogenesis inhibitor, suppresses liver metastasis and invasive growth of colon cancer in mice. Cancer Gene Ther 2004, 11:419-30.

21. Ivics Z, Hackett PB, Plasterk RH, Izsvak Z: Molecular reconstruction of sleeping beauty, a Tc1-like transposon from fish, and its transposition in human cells. Cell 1997, 91:501-10.

22. Aronovich $E L$, Bell JB, Belur $L R$, et al: Prolonged expression of a lysosomal enzyme in mouse liver after sleeping beauty transposon-mediated gene delivery: Implications for non-viral gene therapy of mucopolysaccharidoses. J Gene Med 2007, 9:403-15.

23. Wilber $A$, Linehan $J$, Tian $X$, et al: Efficient and stable transgene expression in human embryonic stem cells using transposon-mediated gene transfer. Stem Cells 2007, 25:2919-27.
24. Xue $X$, Huang $X$, Nodland $S E$, et al: Stable gene transfer and expression in cord blood-derived CD34+ hematopoietic stem and progenitor cells by a hyperactive sleeping beauty transposon system. Blood 2009, 114:1319-30.

25. Yant SR, Meuse L, Chiu W, Ivics Z, Izsvak Z, Kay MA: Somatic integration and long-term transgene expression in normal and haemophilic mice using a DNA transposon system. Nat Genet 2000, 25:35-41.

26. Huang $X$, Guo $H$, Kang J, et al: Sleeping beauty transposon-mediated engineering of human primary T cells for therapy of CD19+ lymphoid malignancies. Mol Ther 2008, 16:580-9.

27. Ohlfest JR, Demorest ZL, Motooka Y, et al: Combinatorial antiangiogenic gene therapy by nonviral gene transfer using the sleeping beauty transposon causes tumor regression and improves survival in mice bearing intracranial human glioblastoma. Mol Ther 2005, 12:778-88.

28. Song J, Kim C, Ochoa ER: Sleeping beauty-mediated suicide gene therapy of hepatocellular carcinoma. Biosci Biotechnol Biochem 2009, 73:165-8.

29. Yokoyama Y, Dhanabal M, Griffioen AW, Sukhatme VP, Ramakrishnan S: Synergy between angiostatin and endostatin: Inhibition of ovarian cancer growth. Cancer Res 2000, 60:2190-6.

30. Raikwar SP, Temm CJ, Raikwar NS, Kao C, Molitoris BA, Gardner TA: Adenoviral vectors expressing human endostatin-angiostatin and soluble Tie2: Enhanced suppression of tumor growth and antiangiogenic effects in a prostate tumor model. Mol Ther 2005, 12:1091-100

31. Ponnazhagan S, Mahendra G, Kumar S, et al: Adeno-associated virus 2mediated antiangiogenic cancer gene therapy: Long-term efficacy of a vector encoding angiostatin and endostatin over vectors encoding a single factor. Cancer Res 2004, 64:1781-7.

32. Scappaticci FA, Smith R, Pathak A, et al: Combination angiostatin and endostatin gene transfer induces synergistic antiangiogenic activity in vitro and antitumor efficacy in leukemia and solid tumors in mice. Mol Ther 2001, 3:186-96.

33. Cui Z, Geurts AM, Liu G, Kaufman CD, Hackett PB: Structure-function analysis of the inverted terminal repeats of the sleeping beauty transposon. J Mol Biol 2002, 318:1221-35.

34. Jain RK, Duda DG, Clark JW, Loeffler JS: Lessons from phase III clinical trials on anti-VEGF therapy for cancer. Nat Clin Pract Oncol 2006, 3:24-40.

35. Gerber HP, Ferrara N: Pharmacology and pharmacodynamics of bevacizumab as monotherapy or in combination with cytotoxic therapy in preclinical studies. Cancer Res 2005, 65:671-80.

36. Chase $\mathrm{L}$ : Clinical use of anti-vascular endothelial growth factor monoclonal antibodies in metastatic colorectal cancer. Pharmacotherapy 2008, 28(11 Pt 2):23S-30S.

37. Ferrara N, Gerber HP, LeCouter J: The biology of VEGF and its receptors. Nat Med 2003, 9:669-76.

38. Ellis LM, Takahashi Y, Liu W, Shaheen RM: Vascular endothelial growth factor in human colon cancer: Biology and therapeutic implications. Oncologist 2000, 5(Suppl 1):11-5.

39. Ellis LM, Hicklin DJ: VEGF-targeted therapy: Mechanisms of anti-tumour activity. Nat Rev Cancer 2008, 8:579-91.

40. Li X, Liu YH, Lee SJ, Gardner TA, Jeng MH, Kao C: Prostate-restricted replicative adenovirus expressing human endostatin-angiostatin fusion gene exhibiting dramatic antitumor efficacy. Clin Cancer Res 2008, 14:291-9.

41. Balciunas D, Ekker SC: Trapping fish genes with transposons. Zebrafish 2005, 1:335-41.

42. Dupuy AJ, Akagi K, Largaespada DA, Copeland NG, Jenkins NA: Mammalian mutagenesis using a highly mobile somatic sleeping beauty transposon system. Nature 2005, 436:221-6.

43. Belur LR, Frandsen $J$, Dupuy $A J$, et al: Gene insertion and long-term expression in lung mediated by the sleeping beauty transposon system. Mol Ther 2003, 8:501-7.

44. Aronovich EL, Bell JB, Khan SA, et al: Systemic correction of storage disease in MPS I NOD/SCID mice using the sleeping beauty transposon system. Mol Ther 2009, 17:1136-44.

45. Kren BT, Unger GM, Sjeklocha L, et al: Nanocapsule-delivered sleeping beauty mediates therapeutic factor VIII expression in liver sinusoidal endothelial cells of hemophilia A mice. J Clin Invest 2009, 119:2086-99.

46. Score PR, Belur LR, Frandsen $J$, et al: Sleeping beauty-mediated transposition and long-term expression in vivo: Use of the LoxP/Cre recombinase system to distinguish transposition-specific expression. $\mathrm{Mol}$ Ther 2006, 13:617-24 
47. Wilber A, Wangensteen KJ, Chen Y: Messenger RNA as a source of transposase for sleeping beauty transposon-mediated correction of hereditary tyrosinemia type I. Mol Ther 2007, 15:1280-1287.

48. Montini E, Held PK, Noll M, et al: In vivo correction of murine tyrosinemia type I by DNA-mediated transposition. Mol Ther 2002, 6:759-69.

49. Liu G, Aronovich EL, Cui Z, et al: Excision of Sleeping Beauty transposons: parameters and applications to gene therapy. J Gene Med 2004, 6:574-83.

50. Bell JB, Aronovich EL, Schreifels JM, et al: Duration of Expression and Activity of Sleeping Beauty Transposase in mouse liver following hydrodynamic DNA delivery. Mol Ther 2010, 18:1796-1802.

51. Martin HA, Zhang W, Müther N, et al: Hyperactive Sleeping Beauty Transposase Enables Persistent Phenotypic Correction in Mice and a Canine Model for Hemophilia B. Mol Ther 2010, 18:1896-1906.

52. Ohlfest JR, Frandsen JL, Fritz $S$, et al: Phenotypic correction and long-term expression of factor VIII in hemophilic mice by immunotolerization and nonviral gene transfer using the Sleeping Beauty transposon system. Blood 2005, 105:2691-8.

53. Wu A, Oh S, Ericson $\mathrm{K}$, et al: Transposon-based interferon gamma gene transfer overcomes limitations of episomal plasmid for immunogene therapy of glioblastoma. Cancer Gene Ther 2007, 14:550-60.

54. Eastman SJ, Baskin KM, Hodges BL, et al: Development of catheter-based procedures for transducing the isolated rabbit liver with plasmid DNA. Hum Gene Ther 2002, 13:2065-77.

55. Yoshino H, Hashizume K, Kobayashi E: Naked plasmid DNA transfer to the porcine liver using rapid injection with large volume. Gene Ther 2006, 13:1696-1702.

56. Fabre JW, Grehan A, Whitehorne M, et al: Hydrodynamic gene delivery to the pig liver via an isolated segment of the inferior vena cava. Gene Ther 2008, 15:452-62.

57. Hackett PB, Urness M, Bell JB, et al: Long-term gene expression after hydrodynamic delivery of Sleeping Beauty transposons to canine liver using balloon catheters. Mol Ther 2010, 18:325.

58. Suda T, Suda K, Liu D: Computer-assisted hydrodynamic gene delivery. Mol Ther 2008, 16:1098-104.

59. Kamimura K, Zhang G, Liu D: Image-guided, intravascular hydrodynamic gene delivery to skeletal muscle in pigs. Mol Ther 2010, 18:93-100.

60. Lutzko C, Kruth S, Abrams-Ogg AC, et al: Genetically corrected autologous stem cells engraft, but host immune responses limit their utility in canine alpha-L-iduronidase deficiency. Blood 1999, 15:1895-905.

61. Schowalter DB, Himeda CL, Winther BL, et al: Implication of interfering antibody formation and apoptosis as two different mechanisms leading to variable duration of adenovirus-mediated transgene expression in immune-competent mice. J Virol 1999, 73:4755-66.

62. Hodges BL, Taylor KM, Joseph MF, et al: Long-term transgene expression from plasmid DNA gene therapy vectors is negatively affected by CpG dinucleotides. Mol Ther 2004, 10:269-78.

63. Multhaup MM, Karlen AD, Swanson DL, et al: Cytotoxicity associated with artemis over-expression after lentiviral vector mediated gene transfer. Hum Gene Ther 2010, 21(7):865-75.

64. Lafreniere R, Rosenberg SA: A novel approach to the generation and identification of experimental hepatic metastases in a murine model. J Natl Cancer Inst 1986, 76:309-322.

65. Belur LR, Mclvor RS, Wilber A: Liver-directed gene therapy using the sleeping beauty transposon system. Methods Mol Biol 2008, 434:267-76.

66. Wilber A, Frandsen JL, Wangensteen KJ, et al: Dynamic gene expression after systemic delivery of plasmid DNA as determined by in vivo bioluminescence imaging. Hum Gene Ther 2005, 16:1325-32.

doi:10.1186/1476-4598-10-14

Cite this article as: Belur et al:. Inhibition of angiogenesis and suppression of colorectal cancer metastatic to the liver using the

Sleeping Beauty Transposon System. Molecular Cancer 2011 10:14.

\section{Submit your next manuscript to BioMed Central and take full advantage of:}

- Convenient online submission

- Thorough peer review

- No space constraints or color figure charges

- Immediate publication on acceptance

- Inclusion in PubMed, CAS, Scopus and Google Scholar

- Research which is freely available for redistribution

Submit your manuscript at www.biomedcentral.com/submit
Biomed Central 